\title{
Inorganic-Organic Hybrid Luminescent Binary Probe for DNA Detection Based on Spin-Forbidden Resonance Energy Transfer
}

\author{
Angel A. Marti ${ }^{\dagger}$, Cindy A. Puckett ${ }^{\ddagger}$, Joanne Dyer ${ }^{\dagger}$, Nathan Stevens ${ }^{\dagger}$, Steffen Jockusch ${ }^{\dagger}$, \\ Jingyue Jull,, , Jacqueline K. Barton $\ddagger$, and Nicholas J. Turro ${ }^{\dagger}, \perp,{ }^{*}$ \\ † Department of Chemistry, Columbia University, New York, NY 10027 \\ ${ }^{\perp}$ Department of Chemical Engineering, Columbia University, New York, NY 10027 \\ ‡ Division of Chemistry and Chemical Engineering, California Institute of Technology, Pasadena, \\ California 91125 \\ I Columbia Genome Center, Columbia University College of Physicians and Surgeons, New York, \\ NY 10032
}

\begin{abstract}
We describe the design of new fluorescent binary probe sensors for DNA detection based on spinforbidden resonance energy transfer (SF-RET). Binary probes consist of a donor and acceptor fluorophores that are attached to two different oligonucleotides and serve as resonance energy transfer (RET) donor-acceptor pair when hybridized to adjacent sites of a target sequence. In the absence of target, excitation of the donor results in fluorescence only from the donor, but when the probes hybridize to target, the fluorophores are brought into close proximity favoring RET, yielding fluorescence mainly from the acceptor fluorophore. These new binary probes use the metal complex $\mathrm{Ru}\left(\mathrm{bpy}^{\prime}\right)(\mathrm{DIP})_{2}{ }^{2+}$ as the energy donor and an organic fluorophore (Cy5) as the energy acceptor. Energy transfer from the MLCT state of the Ru complex to singlet Cy 5 is spin forbidden and produces a delayed fluorescence of Cy5. This paper demonstrate that fluorescence delay of Cy5 can be used to time resolve the emission of the probe from the intense fluorescence background of a model system for cellular background; this provides the reported system to overcome intense autofluorescence, an important and general advantage over "classical" spin-allowed steady-state probes.
\end{abstract}

The design of new fluorescent sensors for DNA has applications in following transcription, ${ }^{1}$ developing sensitive diagnostics, ${ }^{2}$ and monitoring biological processes in the cell. ${ }^{3}$ Among the most effective sensors for DNA detection are binary probes, ${ }^{4}$ which have donor and acceptor fluorophores that are attached to two different oligonucleotides and serve as a resonance energy transfer (RET) donor-acceptor pair when hybridized to adjacent sites of a target sequence. ${ }^{5}$ In the absence of target, excitation of the donor results in fluorescence only from the donor, but when the probes hybridize to target, the fluorophores are brought into close proximity favoring RET, yielding fluorescence mainly from the acceptor fluorophore. Thus the selective hybridization of the probes to a target produces a unique fluorescence signal that can be used for detecting target DNA in vitro and in vivo. ${ }^{3,4}$ Binary probes show target specificity, high sensitivity and reliability and avoid false positive signals. ${ }^{6}$ Nonetheless, the sensitive detection of DNA in living cells is often obscured by a high autofluorescence background. ${ }^{7}$ In this report, we describe the design of new binary probes based upon spin-forbidden resonance energy

njt3@columbia.edu.

Supporting Information Available: Experimental procedures, steady-state and lifetime data and determination of optimum time window for TRE spectra. This material is available free of charge via the Internet at http://pubs.acs.org. 
transfer (SF-RET). These probes allow sensitive fluorescence detection of DNA in highly fluorescent cell media.

We earlier reported the utilization of long-lived pyrene binary probes that show monomer emission $(390 \mathrm{~nm})$ when free in solution and excimer emission $(480 \mathrm{~nm})$ when bound to target. ${ }^{8}$ The long fluorescence lifetime of these probes allowed the pyrene signal to be time-resolved from short-lived emission that is typical of cellular autofluorescence. Notwithstanding the inherent advantages of the pyrene probe, its UV excitation $(350 \mathrm{~nm})$ and its relatively short lifetime in aqueous solution ( $40 \mathrm{~ns}$ ) challenged us to design probes with properties suited to detect DNA targets in living cells. To meet this challenge, we have now utilized the metal complex $\mathrm{Ru}\left(\mathrm{bpy}^{\prime}\right)(\mathrm{DIP})_{2}{ }^{2+}$ as the energy donor and an organic fluorophore (Cy5) as the energy acceptor (Figure 1).

A characteristic of ruthenium(II) polypyridyl complexes is their long lived luminescent metal to ligand charge transfer (MLCT) excited states. ${ }^{9}$ Energy transfer from the MLCT state to singlet Cy5 is spin forbidden (SF-RET). Although SF-RET occurs at a slower rate, the very long emission lifetime of the Ru-complex $(1.8 \mu \mathrm{s})$ easily compensates, leading to high RET efficiency. ${ }^{10}$

Oligonucleotide probes were synthesized to examine SF-RET. Ru(bpy')(DIP) $2^{2+}$ tethered to the $5^{\prime}$-end was used as the energy donor and oligonucleotides containing $3^{\prime}$-tethered Cy5 were used as acceptors; three of these acceptor oligonucleotides were synthesized with variable sequences to produce different distances between the $\mathrm{Ru}$ and $\mathrm{Cy} 5$ probes when hybridized to target. ${ }^{11}$ Figure 2 shows the luminescence spectra of one probe set with and without target by steady-state luminescence and time resolved emission (TRE). When the probes are free in solution, only emission from the ruthenium complex is observed $\left(\lambda_{\mathrm{Ex} .}=440 \mathrm{~nm}\right)$. In the presence of target, $\mathrm{Ru}\left(\mathrm{bpy}^{\prime}\right)(\mathrm{DIP})_{2}{ }^{2+}$ and $\mathrm{Cy} 5$ are brought into close proximity, a condition favorable for RET, and mainly Cy5 emission is observed.

The steady-state luminescence spectra for all three oligonucleotide sets are similar (Supporting Information). However, time-resolved lifetime measurements show important differences. The lifetime of Cy5 is ca. $2 \mathrm{~ns}$ when free in solution, but seems to increase in the presence of target with excitation of Ru. For the three sets, with increasing probe separation, the lifetimes are 45, 46, and $69 \mathrm{~ns}$. The delayed fluorescence of Cy5 results from the slow SF-RET rate constant, which decreases with longer distance. Figure $2 \mathrm{~b}$ shows the TRE spectrum for one oligonucleotide set. ${ }^{12,13}$ The TRE spectrum is similar to its steady-state counterpart with a slight improvement in the signal to background ratio (S/B). ${ }^{14}$ This improvement is attributed to the near depopulation of directly excited $\mathrm{Cy} 5$ on the time scale of the TRE measurement.

To mimic cellular autofluorescence, we tested the probes in Dulbecco's cell medium, adding the fluorophore Red-X to further intensify the medium's fluorescence. ${ }^{15}$ The steady-state spectrum is dominated by the brightly fluorescent background and changes only modestly upon the addition of target (Figure 2c). The S/B is reduced compared to the probes in buffer (Figure $2 \mathrm{a})$ from 14 to 2.5. In comparison, the TRE spectrum (Figure $2 \mathrm{~d}$ ), even in the presence of strongly fluorescent cell medium, shows a spectral profile that closely resembles that in buffer. Integrating the emission signal from 59-77 ns allows measurement of a TRE spectrum after most of the background fluorescence has decayed. Although some background remains, it is dramatically reduced by TRE, with the S/B increasing from 2.5 to 10 .

To further demonstrate the advantages of SF-RET probes over standard spin-allowed RET (SA-RET) probes, we replaced the Ru complex with Alexa 488, a singlet energy donor (Figure 1). ${ }^{4}$ Figure 3 a shows that, as expected, the Alexa 488 fluorescence intensity decreases and the Cy5 fluorescence intensity increases when the target is added as a result of RET. The TRE spectrum successfully reproduces the fluorescence emission profiles in buffer, but fails in 
Dulbecco's cell medium supplemented with RedX (Figure 3b, d). The background fluorescence of the cell medium has a fluorescence lifetime similar to that of the probes ( $2 \mathrm{~ns})$, making impossible the discrimination of the RET signal from background using TRE.

Figure 4 shows the direct comparison for the two systems (SF versus SA-RET). As is evident, the longer time scale for SF-RET permits sufficient time for background autofluorescence to decay.

In summary, we have designed, synthesized and successfully demonstrated the utility of SFRET to target DNA using a hybrid inorganic-organic probe set with a ruthenium complex as donor and Cy5 as acceptor. With TRE, the delayed fluorescence of the Cy5 acceptor due to SF-RET can be employed to distinguish target signal, from intense, but short-lived background emission such as cell autofluorescence. Additionally, Ru complexes possess tunable absorption and emission profiles, by using different ligands, an advantage for use with fluorescence microscopes. SF-RET may be useful for the detection of DNA and RNA in highly fluorescent media in vitro and in vivo. Further studies are under progress to evaluate the effectiveness of these probes inside living cells.

\section{Supplementary Material}

Refer to Web version on PubMed Central for supplementary material.

\section{Acknowledgments}

This work was supported by the Center of Excellence in Genomic Science Grant P50 HG002806 from the NIH and by the NSF under grant NSF CHE-04-15516. J.K.B. thanks the NIH (GM33309) for financial support.

\section{References}

1. Marras SAE, Tyagi S, Kramer FR. Clin Chim Acta 2006;363:48-60. [PubMed: 16111667]

2. Mergny JL, Boutorine AS, Garestier T, Belloc F, Rougee M, Bulychev NV, Koshkin AA, Bourson J, Lebedev AV, Valeur B, Thuong NT, Helene C. Nucleic Acids Res 1994;22:920-928. [PubMed: 8152922]

3. Tsuji A, Koshimoto H, Sato Y, Hirano M, Seilida Y, Kondo S, Ishibashi K. Biophys J 2000;78:32603274. [PubMed: 10828002]

4. Marti AA, Li X, Jockusch S, Stevens N, Li Z, Raveendra B, Kalachikov S, Morozova I, Russo JJ, Akins DL, Ju J, Turro NJ. Tetrahedron. 200710.1016/j.tet.2006.1008.1109

5. Cardullo RA, Agrawal S, Flores C, Zamecnik PC, Wolf DE. Proc Natl Acad Sci U S A 1988;85:87908794. [PubMed: 3194390]

6. Marti AA, Jockusch S, Stevens N, Ju J, Turro NJ. Acc Chem Res. 2007accepted

7. Mosiman VL, Patterson BK, Canterero L, Goolsby CL. Cytometry 1997;30:151-156. [PubMed: 9222101]

8. Marti AA, Li X, Jockusch S, Li Z, Raveendra B, Kalachikov S, Russo JJ, Morozova I, Puthanveettil SV, Ju J, Turro NJ. Nucleic Acids Res 2006;34:3161-3168. [PubMed: 16769776]

9. Kalyanasundaram, K. Photochemistry of Polypyridine and Porphyrin Complexes. Academic Press; New York: 1992. p. 106-164.

10. Turro, NJ. Modern molecular photochemistry. University science books; Sausalito, CA: 1991. p. 338-339.

11. Ru-probe/Alexa-probe used here: 5'-AAG TTG ATC AAG TTG GT-Ru (or Alexa488)-3'; Cy5Probe-3: 5'-Cy5-TTC ACT GGA TGA-3'. Other Cy5 probe sequences are in Supporting Information. For synthesis see Holmlin RE, Dandliker PJ, Barton JK. Bioconjugate Chem 1999;10:11221130.1130 Details and characterization are in Supporting Information.

12. The procedure to determine the optimum integration time window is described in Supporting Information. 
13. Cy5-Probe-3 was selected for TRE since it showed the greatest degree of delayed Cy5 fluorescence. 14. $\mathrm{S} / \mathrm{B}=\left(\mathrm{Em}_{667}\right.$ with target $/ \mathrm{Em}_{667}$ without target $) /\left(\mathrm{Em}_{610}\right.$ with target/Em 610 without target $)$

15. Yang CJ, Jockusch S, Vincens M, Turro NJ, Tan W. Proc Natl Acad Sci U S A 2005;102:1727817283. [PubMed: 16301535] 


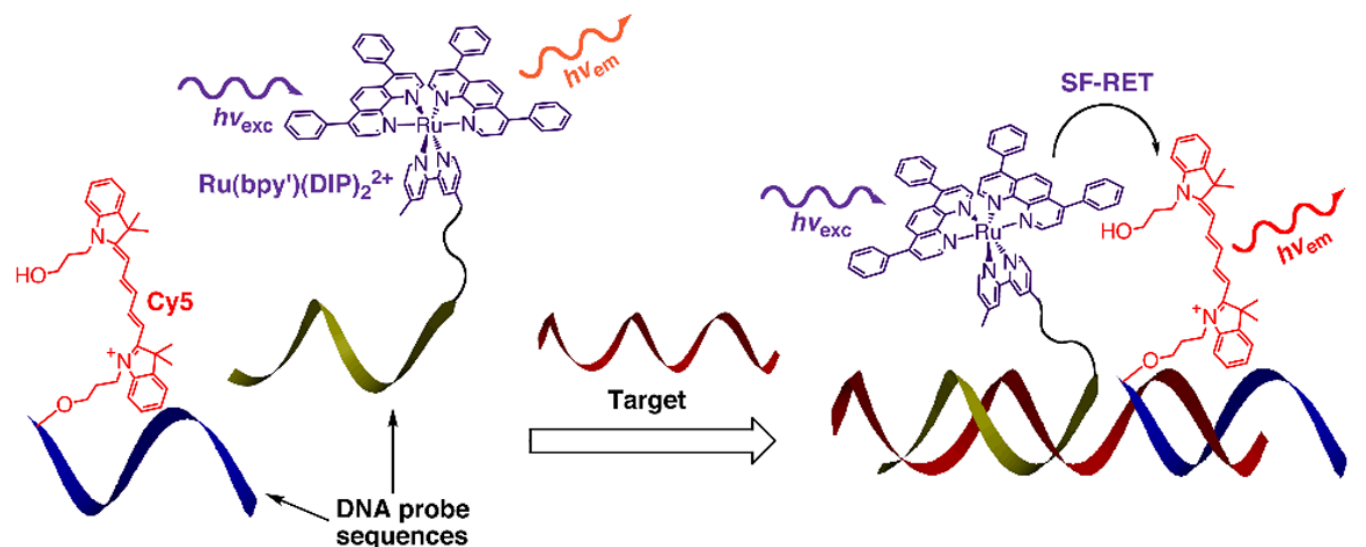

(b) Spin Allowed RET Probes

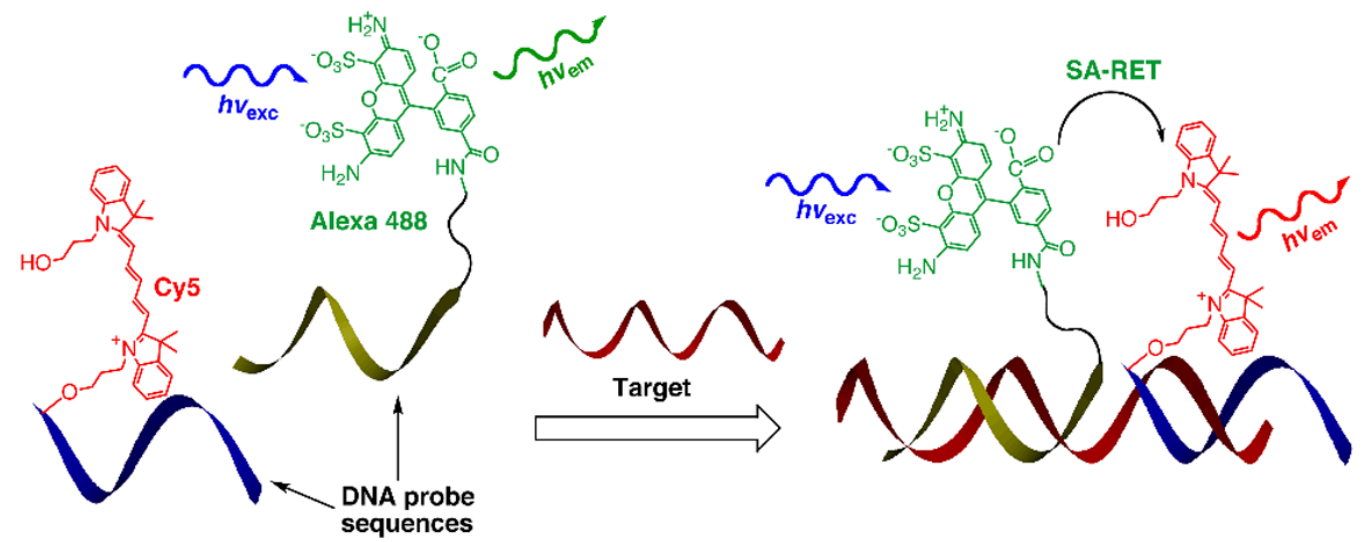

Figure 1.

(a) Spin-forbidden and (b) spin-allowed binary probes. 
(a)

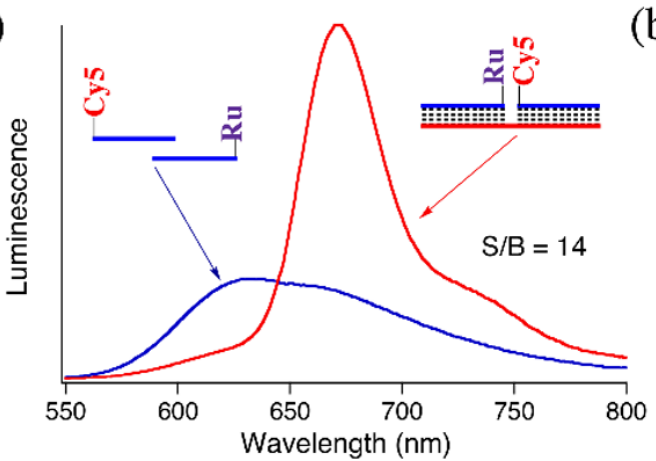

(c)

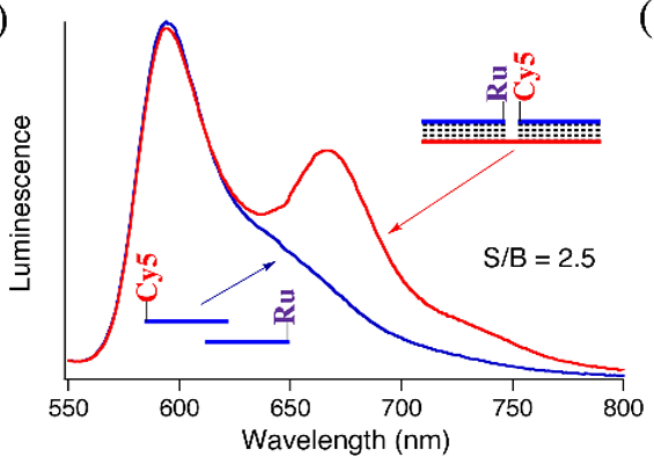

(b)

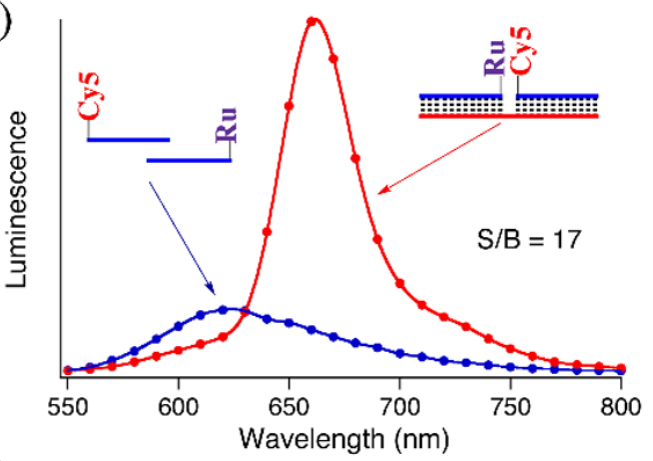

(d)

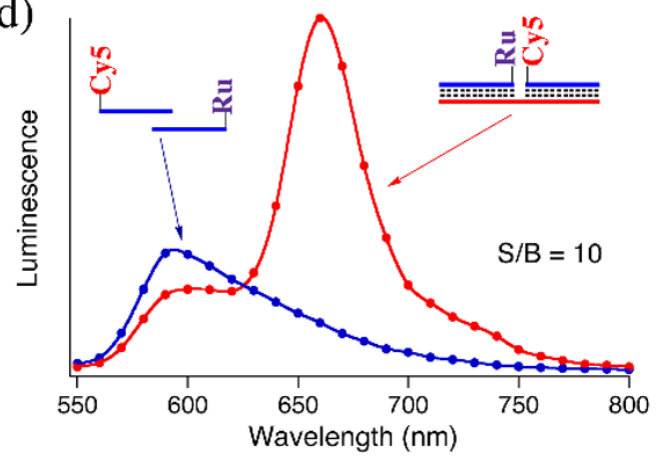

Figure 2.

(a) Steady-state luminescence spectra and (b) TRE (59-77 ns after the excitation pulse) of Ruprobe and Cy5-probe in buffer with (-) and without (-) target. (c) Steady-state luminescence spectra and (d) TRE (59-77 ns after the excitation pulse) of Ru-probe and Cy5-probe in RedXcontaining cell medium with (_) and without (_) target. 
(a)

(c)

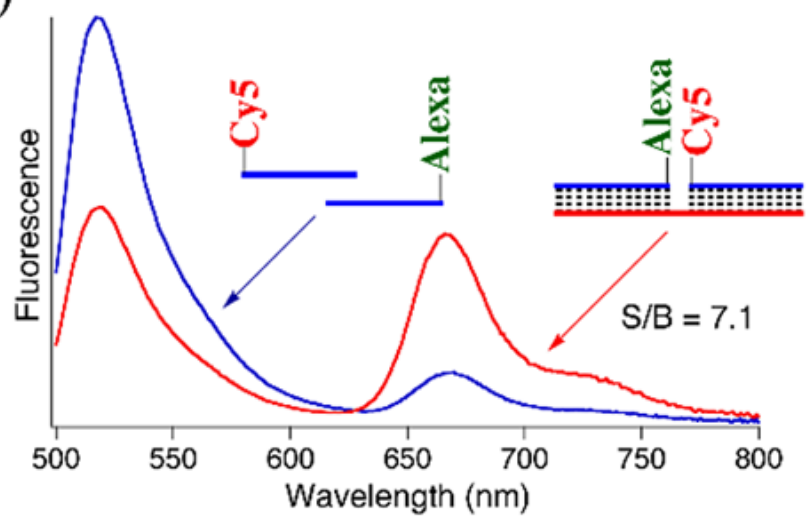

(b)

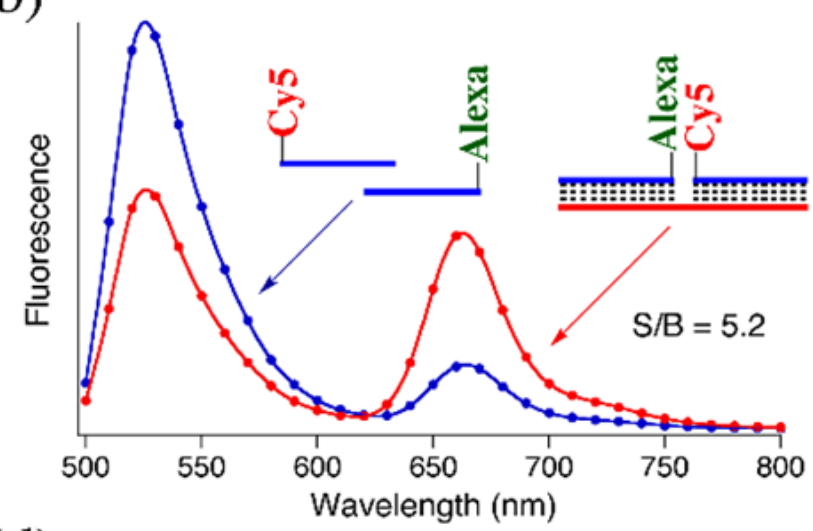

(d)

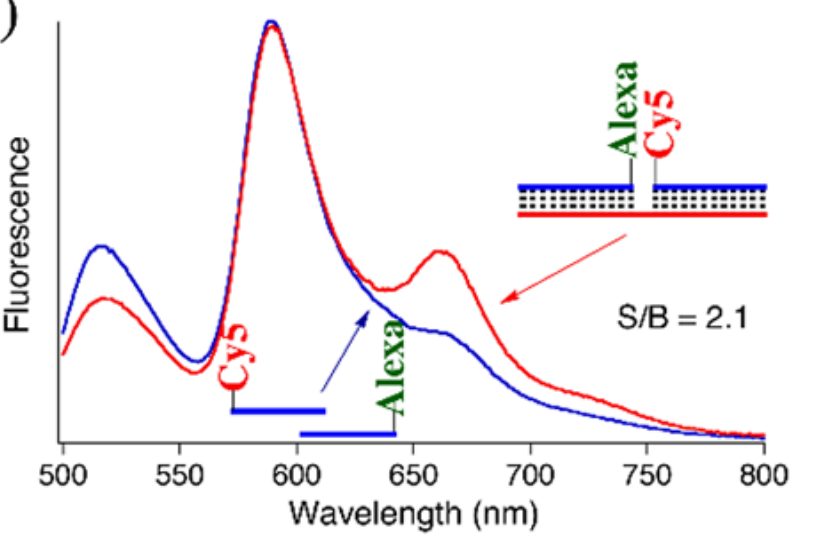

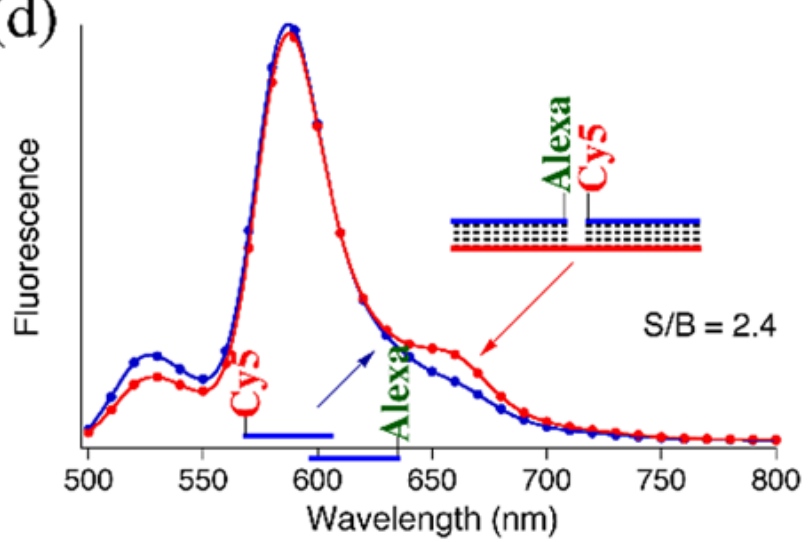

Figure 3.

(a) Steady-state luminescence spectra and (b) TRE (1.0-6.2 ns after the excitation pulse) of Alexa 488-probe and Cy5-probe in buffer with (-) and without (-) target. (c) Steady-state luminescence spectra and (d) TRES (1.0-6.2 ns after the excitation pulse) of Alexa 488-probe and Cy5-probe in RedX-containing cell medium with (-) and without (-) target. 


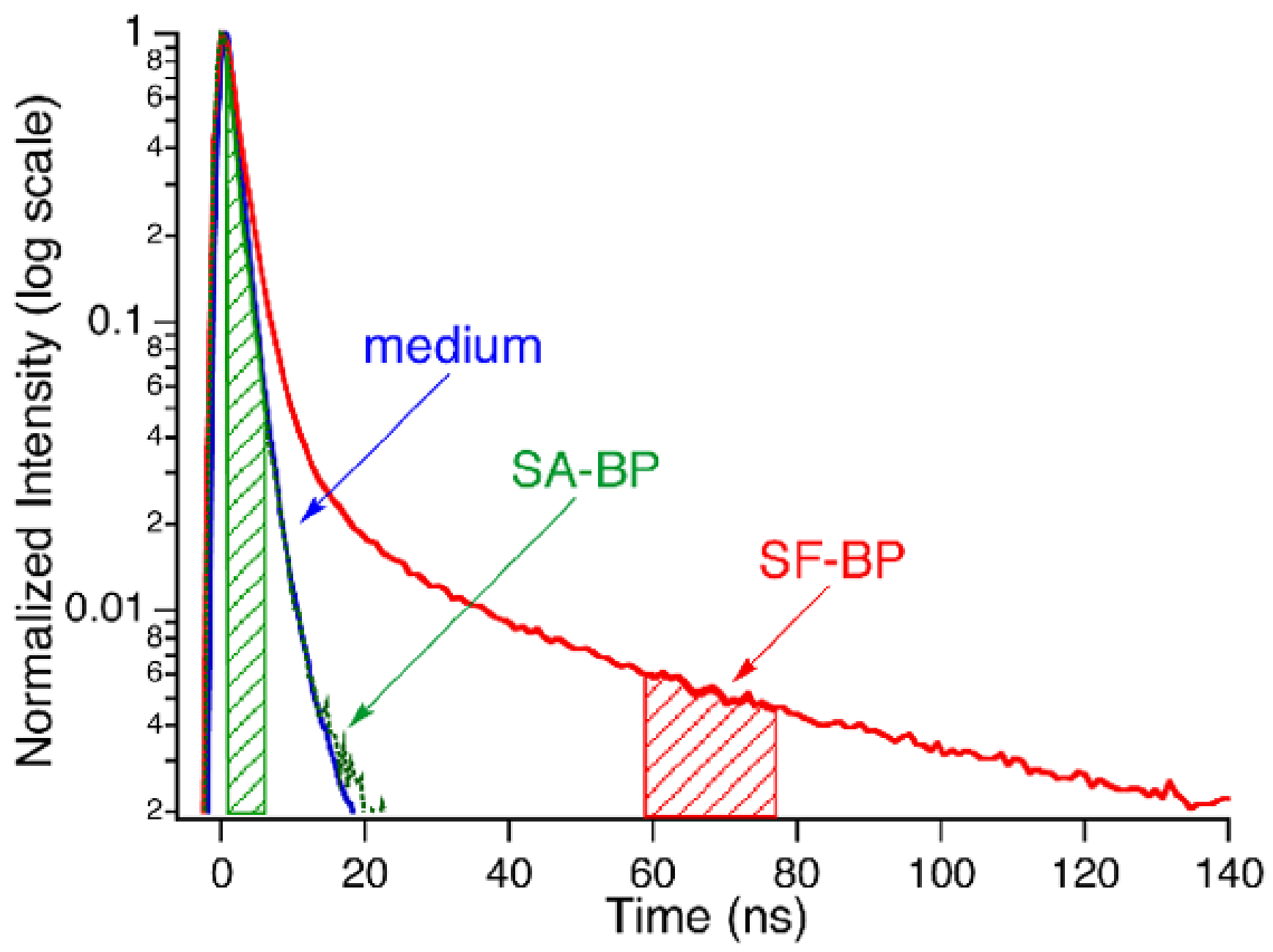

Figure 4.

Fluorescence decay traces of the spin-allowed (...), spin-forbidden (_) binary probes, and medium (-) showing the optima integration time windows. 